\title{
Consensuability Conditions of Multi Agent Systems with Varying Interconnection Topology and Different Kinds of Node Dynamics
}

\author{
Dr. Sabato Manfredi \\ Faculty of Engineering, University of Naples Federico II, Via Claudio 21, Napoli 80120.
}

Italy

\section{Introduction}

Many systems in nature and of practical interest can be modeled as large collections of interacting subsystems. Such systems are referred as "Multi Agent Systems" (briefly MASs) and some examples include electrical power distribution networks (P. Kundur, 1994), communication (F. Paganini, 2001), and collections of vehicles traveling in formation (J.K. Hedrick et al., 1990). Several practical issues concern the design of decentralized controllers and the stability analysis of MASs in the presence of uncertainties in the subsystem interconnection topology (i.e. due in practical applications to failures of transmission lines). The analysis and control of collections of interconnected systems have been widely studied in the literature. Early work on stability analysis and decentralized control of large-scale interconnected systems is found in (D. Limebeer \& Y.S. Hung, 1983; A. Michel \& R. Miller, 1977; P.J. Moylan \& D.J. Hill, 1978; Siljak, 1978; J.C. Willems, 1976). Some of the more widely notable stability criteria are based on the passivity conditions (M. Vidyasagar, 1977) and on the well-known notion of connective stability introduced in (Siljak, 1978).

More recently, MASs have appeared broadly in several applications including formation flight, sensor networks, swarms, collective behavior of flocks (Savkin, 2004; C.C. Cheaha et al., 2009; W. Ren, 2009) motivating the recent significative attention of the scientific community to distributed control and consensus problems (i.e. (R.O. Saber \& R. Murray, 2004; Z. Lin et al., 2004; V. Blondel et al., 2005; J. N. Tsitsiklis et al., 1986)). One common feature of the consensus algorithm is to allow every agent automatically converge to a common consensus state using only local information received from its neighboring agents. "Consensusability" of MASs is a fundamental problem concerning with the existence conditions of the consensus state and it is of great importance in both theoretical and practical features of cooperative protocol (i.e. flocking, rendezvous problem, robot coordination). Results about consensuability of MASs are related to first and second order systems and are based on the assumption of jointly-connected interaction graphs (i.e. in (R.O. Saber \& R. Murray, 2004; J. N. Tsitsiklis et al., 1986)). Extension to more general linear MASs whose agents are described by LTI (Linear Time Invariant) systems can be found in (Tuna, 2008) where the closed-loop MASs were shown to be asymptotic consensus stable if the topology had a spanning tree. In (L. Scardovi \& R. Sepulchre, 2009) it is investigated the synchronization of a 
network of identical linear state-space models under a possibly time-varying and directed interconnection structure. Many investigations are carried out when the dynamic structure is fixed and the communication topology is time varying (i.e. in (R.O. Saber \& R. Murray, 2004; W. Ren \& R. W. Beard, 2005; Ya Zhanga \& Yu-Ping Tian, 2009)). One of main appealing field of research is the investigation of the MASs consensusability under both the dynamic agent structure and communication topology variations. In particular, it is worth analyzing the joint impact of the agent dynamic and the communication topology on the MASs consensusability. The aim of the chapter is to give consensusability conditions of LTI MASs as function of the agent dynamic structure, communication topology and coupling strength parameters. The theoretical results are derived by transferring the consensusability problem into the robust stability analysis of LTI-MASs. Differently from the existing works, here the consensuability conditions are given in terms of the adjacency matrix rather than Laplacian matrix. Moreover, it is shown that the interplay among consensusability, node dynamic and topology must be taken into account for MASs stabilization: specifically, consensuability of MASs is assessed for all topologies, dynamic and coupling strength satisfying a pre-specified bound. From the practical point of view the consensuability conditions can be used for both the analysis and planning of MASs protocols to guarantee robust stability for a wide range of possible interconnection topologies, coupling strength and node dynamics. Also, the number of subsystems affecting the overall system stability is taken into account as it is analyzed the robustness of multi agent systems if the number of subsystems changes. Finally, simulation examples are given to illustrate the theoretical analysis.

\section{Problem statement}

We consider a network composed of linear systems interconnected by a specific topological structure. The dynamical system at each node is of $\mathrm{m}$-th order and described by the matrices $(A, B, C)$. Let $G(V, E, U)$ be a directed weighted graph (digraph) with the set of nodes $V=1 . . n$, set of edges $E \subseteq n \times n$, and the associated weighted adjacency matrix $U=\left\{u_{i j}\right\}$ with $u_{i j}>0$ if there is a directed edge of weight $u_{i j}$ from vertex $\mathrm{j}$ (node parent) into vertex $\mathrm{i}$ (node child). The linear systems are interconnected by a directed weighted graph $G(V, E, U)$. Each node dynamical is described by:

$$
\begin{aligned}
& \dot{x}_{i}(t)=A x_{i}(t)+B v_{i}(t) \\
& y_{i}(t)=C x_{i}(t)
\end{aligned}
$$

with $v_{i}(t)$ is the input to the $\mathrm{i}-\mathrm{th}$ node of the form

$$
v_{i}(t)=\sum_{j=1}^{n} u_{i j} y_{j}(t) .
$$

In this way, each node dynamic is influenced by the sum of its neighbors' outputs. This yields to the MAS network equation:

$$
\dot{x}_{i}(t)=A x_{i}(t)+\sum_{j=1}^{n} u_{i j} B C x_{j}(t)
$$

with $1 \leq i \leq n$, and its compact form:

$$
\dot{x}(t)=A_{g} x(t)
$$


with $A_{g}=\left(I_{n} \otimes A\right)+(U \otimes B C)$, with $\otimes$ denotes the matrix Kronecker product. Notice that the above equation can be associated to the main model used in the literature for describing the synchronization phenomena, energy distribution, tanks network (e.g. in (R. Cogill \& S. Lall, 2004)). Moreover the system at each node can be MIMO or SISO type, and the matrix product $B C$ takes into account the coupling strength and the coupling interaction among the state system variables. Observing the MAS model (3) we point out as the overall network dynamic is affected by the node system dynamic matrix $A$, the coupling matrix $B C$, and by the adjacency matrix $U$ of the topological structure.

Consider a network with $n$ agents whose topology information exchange is described by a graph $G(V, E, U)$ and let $x_{i}$ the state of agent-node i-th, consensus corresponds to the network condition such that the state of the agents as a whole asymptotically converges to an equilibrium state with identical elements (i.e. $x_{i}=x_{j}$ for all $\left.(i, j) \in n \times n\right)$. The common value $\bar{x}$ is named consensus value. Consensusability of MASs is a fundamental problem concerning with the conditions for assessing network consensus equilibrium. Under the assumption of the existence of a network equilibrium, then consensuability deals with the research of analytical conditions such that the network equilibrium corresponds to a consensus state. In this way, without loss of generality, the consensuability problem can be reduced to the problem of assessing stabilization conditions of the MAS network (3) with respect to the 0 equilibrium point (i.e. $x_{i}=x_{j}=0$ for all $(i, j) \in n \times n$ ).

Hence, we are interested in solving the following problem:

Problem Given a multi agent network described by (3), to determinate the MAS consensuability conditions as function of node dynamic, topology and coupling strength.

Specifically, consensuability of MASs is assessed for all topologies, dynamic and coupling strength satisfying a pre-specified bound.

In the follows we will present analytical conditions for solving the above Problem.

\section{Conditions for MASs consensuability}

Before of presenting the MASs consensuability conditions of (3), we have to recast the eigenvalues set $\sigma\left(A_{g}\right)$ of MAS network dynamic matrix $A_{g}$.

Lemma 1 Let $\sigma(U)=\left\{\mu_{i}\right\}$ the eigenvalues set of the adjacency matrix $U, \sigma\left(A_{g}\right)$ the eigenvalues set of the MAS dynamical matrix $A_{g}$, then results: $\sigma\left(A_{g}\right)=\bigcup_{i} \sigma\left(A+\mu_{i} B C\right)$ for all $1 \leq i \leq n$.

Proof Let $J$ the Jordan canonical form of $U$, then it exists a similarity matrix $S$ so that $J=$ $S^{-1} U S$. Hence $S \otimes I_{n}$ is a similarity matrix for the matrices $I_{m} \otimes A+U \otimes B C$ and $I_{m} \otimes A+$ $J \otimes B C$. From the Kronecker product (Horn R.A. \& Johnson C.R., 1995) results:

$$
\begin{array}{r}
\left(S \otimes I_{n}\right)^{-1}\left(I_{m} \otimes A+U \otimes B C\right)\left(S \otimes I_{n}\right)= \\
\left(S^{-1} \otimes I_{n}\right)\left(I_{m} \otimes A+U \otimes B C\right)\left(S \otimes I_{n}\right)= \\
\left(I_{m} \otimes A\right)+\left(S^{-1} U S \otimes B C\right)=\left(I_{m} \otimes A\right)+(J \otimes B C)
\end{array}
$$

with $J$ being an upper triangular matrix with $I_{m} \otimes A+J \otimes B C$ as upper triangular block matrix. Hence the eigenvalues of the matrix $I_{m} \otimes A+J \otimes B C$ are the union of the eigenvalues of the block matrix on the diagonal. 
From the above Lemma 1, the eigenvalues of the MAS dynamic matrix $A_{g}$ are explicitly function of those of the matrix $A+\mu_{i} B C$, for all $i$. So we can decouple the effects of topology structure (by $\mu_{i}$ ), the coupling strength $B C$ and node dynamic $A$ on the overall stability of the MAS. This can be used for giving stability MAS condition as function of topology structure, node dynamic and coupling strength as shown by the following Theorem 1:

Theorem 1 Let the MAS composed of $n$ identical MIMO system of order m-th and interconnected by the digraph $G=(V, E, U)$ with adjacency matrix $U$, with eigenvalues $\mu_{1} \leq \mu_{2} \leq \ldots \mu_{n}$. If the node dynamic matrix $A=\left\{a_{i j}\right\}$ and the coupling matrix $B C=\left\{c_{i j}\right\}$ fulfill the conditions:

$$
\begin{aligned}
a_{i i}+\mu_{k} c_{i i} & \leq 0 \\
\left|a_{i i}+\mu_{k} c_{i i}\right| & \geq \sum_{j \neq i}\left|a_{i j}+\mu_{k} c_{i j}\right|
\end{aligned}
$$

$\forall i=1,2, \ldots, m$ and $\forall k=1,2, \ldots, n$, then the MAS (3) is stable.

Proof If the conditions (5) hold, then all eigenvalues of the matrix

$$
A+\mu_{k} B C=\left(\begin{array}{cccc}
a_{11}+\mu_{k} c_{11} & a_{12}+\mu_{k} c_{12} & \ldots & a_{1 m}+\mu_{k} c_{1 n} \\
a_{21}+\mu_{k} c_{21} & a_{22}+\mu_{k} c_{22} & \ldots & a_{2 m}+\mu_{k} c_{2 m} \\
\vdots & \vdots & \vdots & \ddots \\
a_{m 1}+\mu_{k} c_{m 1} & a_{m 2}+\mu_{k} c_{m 2} & \ldots & a_{m m}+\mu_{k} c_{m m}
\end{array}\right),
$$

$\forall k=1,2, \ldots, n$, are located in a convex set in the left complex half plane as result by the application of the Gershgorin's circle theorem (Horn R.A. \& Johnson C.R., 1995). Hence, by Lemma 1, the MAS is stable.

The previous Theorem 1 easily yields to the following corollaries.

Corollary 1 Let the MAS composed of $n$ identical MIMO system of order 2 and interconnected by the digraph $G=(V, E, U)$, with adjacency matrix $U$ with eigenvalues $\mu_{1} \leq \mu_{2} \leq \ldots \mu_{n}$. If the node dynamic matrix $A=\left\{a_{i j}\right\}$ and the coupling matrix $B C=\left\{c_{i j}\right\}$ with $c_{i j} \geq 0, i, j=1,2$, fulfill the conditions:

$$
\begin{aligned}
& a_{i j} \geq-c_{i j} \mu_{1} \\
& a_{i i} \leq-a_{i j}-\left(c_{i i}+c_{i j}\right) \cdot \mu_{n}
\end{aligned}
$$

or

$$
\begin{aligned}
& a_{i i} \leq-c_{i i} \mu_{n} \\
& a_{i j} \leq-c_{i j} \mu_{n} \\
& a_{i i} \leq a_{i j}+\left(c_{i j}-c_{i i}\right) \cdot \mu_{1},
\end{aligned}
$$

$i, j=1,2$, then the MAS (3) is stable. 
Because the adjacency matrix $U$ of a graph has both positive and negative eigenvalues, the conditions (6) and (7) implicitly imply the assumption that the single system at the node is stable. In this way, as expected, we derive that it is not possible to stabilize a network of instable systems by acting only on the topological structure. Given a specified node dynamic, coupling strength and bound on the adjacency matrix $U$, by conditions (6) and (7) we can assess MAS stability. Moreover, the MAS robustness with respect to varying switching topology can be dealt by considering the span of the eigenvalue of the admissible structure topologies. As we will show in the follows, it is possible easily to evaluate the eigenvalues of $A_{g}$, given the eigenvalues of $U$ in some simple and representative cases of interest.

Corollary 2 Let the MAS composed of $n$ identical MIMO system of order 1 and interconnected by the digraph $G=(V, E, U)$, with adjacency matrix $U$ with eigenvalues $\mu_{1} \leq \mu_{2} \leq \ldots \mu_{n}$. If the node dynamic matrix $A=a$ and the coupling matrix $B C=c$ fulfill the conditions:

$$
\begin{array}{ll}
a \leq-c \cdot \mu_{n} & \text { if } \quad c \geq 0 \\
a \leq-c \cdot \mu_{1} & \text { if } \quad c<0
\end{array}
$$

then the MAS (3) is stable.

The Corollary 2 reduces the analytical result of Theorem 1 to the case of the consensus of integrator (R.O. Saber \& R. Murray, 2004) with coupling gain c. Smaller c, higher is the degree of robustness of the network to the slower node dynamic. In the opposite, higher $c$ reduces the stability margin of the MAS. Finally, for a fixed dynamic at the node, the maximum admissible coupling strength $c$ depends on the maximum and minimum eigenvalues of the adjacency matrix:

$$
\begin{array}{ll}
c \leq-\frac{a}{\mu_{n}} & \text { if } \quad c \geq 0 \\
c \geq-\frac{a}{\mu_{1}} \quad \text { if } \quad c<0 .
\end{array}
$$

Corollary 3 Let the MAS of $n$ identical MIMO system of the $m$-th order, interconnected by the digraph $G=(V, E, U)$, with adjacency matrix $U$ with eigenvalues $\mu_{1} \leq \mu_{2} \leq \ldots \mu_{n}$. If the node dynamic matrix $A=\left\{a_{i j}\right\}$ and the coupling matrix $B C=\left\{c_{i j}\right\}$ are both upper or lower triangular matrix and fulfill the conditions:

$$
\begin{array}{lll}
a_{i i} \leq-c_{i i} \cdot \mu_{n} & \text { if } \quad c_{i i} \geq 0 \\
a_{i i} \leq-c_{i i} \cdot \mu_{1} & \text { if } \quad c_{i i}<0,
\end{array}
$$

then the MAS (3) is stable. 


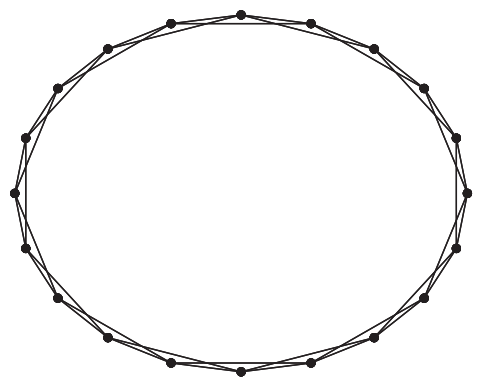

(a)

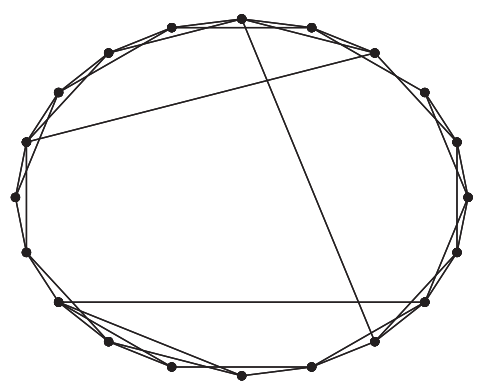

(b)

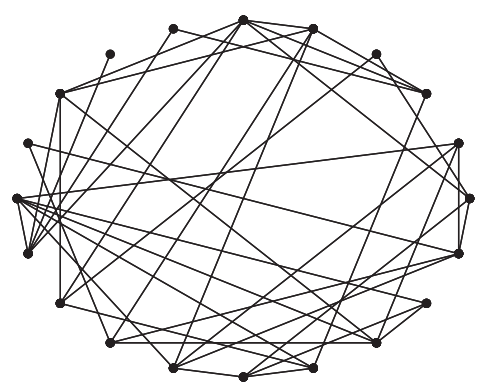

(c)

Fig. 1. Procedure of redirectioning of links in a regular network (a) with increasing probability $p$. As $p$ increases the network moves from regular (a) to random (c), becoming small world (b) for a critical value of $p . \mathrm{n}=20, \mathrm{k}=4$

Notice that if $B C=c \cdot I_{n},(10)$ and (11) become:

$$
\begin{gathered}
c \leq-\frac{\min _{i}\left|a_{i i}\right|}{\mu_{n}} \quad \text { If } \quad c_{i i} \geq 0 \\
c>-\frac{\min _{i}\left|a_{i i}\right|}{\mu_{1}} \quad \text { If } \quad c_{i i}<0
\end{gathered}
$$

and hence the stability of MAS is explicitly given as function of the network slowest node dynamic.

Now we would like to point out the case of undirected topology with symmetric adjacency matrix $U$. If we assume $A$ and $B C$ being symmetric, then $A_{g}$ is symmetric with real eigenvalue. Moreover from the field value property (Horn R.A. \& Johnson C.R., 1995), let $\sigma(A)=\left\{\alpha_{j}\right\}$ and $\sigma(B C)=\left\{v_{j}\right\}$ the eigenvalues set of $A$ and $B C$, then the eigenvalues of $A+\mu_{i} B C$ are in the interval $\left[\min _{j}\left\{\alpha_{j}\right\}+\mu_{i} \min _{j}\left\{v_{j}\right\}, \max _{j}\left\{\alpha_{j}\right\}+\mu_{i} \max _{j}\left\{v_{j}\right\}\right]$, for every $1 \leq i \leq n, 1 \leq j \leq m$. In this way, there is a bound need to be satisfied by the topology structure, node dynamic and coupling matrix for MAS stabilization. 
In the literature, the MAS consensuability results have been given in terms of Laplacian matrix properties. Here, differently, we have given bounds as function of the adjacency matrix features. Anyway we can use the results on the Laplacian eigenvalue for recasting the bounds given on the adjacency matrix. To this aim, defined the degree $d_{i}$ of $\mathrm{i}$-th node of an undirected graph as $\sum_{j} u_{i j}$, the Laplacian matrix is defined as $L=D-U$ with $D$ is the diagonal matrix with the degree of node $\mathrm{i}-\mathrm{th}$ in position $\mathrm{i}-t h$. Clearly $L$ is a zero row sums matrix with non-positive off-diagonal elements. It has at least one zero eigenvalue and all nonzero eigenvalues have nonnegative real parts. So $U=D-L$ and being the minimum and maximum Laplacian eigenvalues respectively bounded by 0 and the highest node degree, we have:

Lemma 2 Let $U$ the adjacency matrix of undirected and connected graph $G=(V, E, U)$, with eigenvalues $\mu_{1} \leq \mu_{2} \leq \ldots \leq \mu_{n}$, then results:

$$
\begin{aligned}
& \mu_{1}(U) \geq \min _{i} d_{i}-\min \left(\max _{k, j}\left\{d_{k}+d_{j}:(k, j) \in E(G)\right\}, n\right) \\
& \mu_{n}(U) \leq \max _{i} d_{i}
\end{aligned}
$$

Proof Easily follows from the Laplacian eigenvalues bound and the field value property (Horn R.A. \& Johnson C.R., 1995).

\section{Simulation validation}

In the follows we will present a variety of simulations to validate the above theoretical results under different kinds of node dynamic and network topology variations. Specifically the MAS topology variations have been carried out by using the well known Watts-Strogats procedure described in (Watts \& S. H. Strogatz, 1998). In particular, starting from the regular network topology $(p=0)$, by increasing the probability $p$ of rewiring the links, it is possible smoothly to change its topology into a random one $(p=1)$, with small world typically occurring at some intermediate value. In so doing neither the number of nodes nor the overall number of edges is changed. In Fig. 1 it shown the results in the case of MAS of 20 nodes with each one having $k=4$ neighbors.

Among the simulation results we focus our attention on the maximum and minimum eigenvalues of the matrixes $U$ (i.e. $\mu_{n}$ and $\mu_{1}$ ) and $A_{g}$ (i.e. $\lambda_{M}$ and $\lambda_{m}$ ) and their bounds computed by using the results of the previous section. In particular, by Lemma 2 , we convey the bounds on $U$ eigenvalues in bounds on $A_{g}$ eigenvalues suitable for the case of time varying topology structure. We assume in the simulations the matrices $A$ and $B C$ to be symmetric. In this way, if $U$ eigenvalues are in $\left[v_{1}, v_{2}\right]$, let $\sigma(A)=\left\{\alpha_{i}\right\}, \sigma(B C)=\left\{v_{i}\right\}$, the eigenvalues of $A g$ will be in the interval $\left[\min _{i} \alpha_{i}+\min _{j}\left\{v_{1} v_{j}, v_{2} v_{j}\right\}, \max _{i} \alpha_{i}+\max _{j}\left\{v_{1} v_{j}, v_{2} v_{j}\right\}\right]$ for $i, j=1,2, \ldots, n$. Notice that, known the interval of variation $\left[v_{1}, v_{2}\right]$ of the eigenvalues set of $U$ under switching topologies, we can recast the conditions (8), (9), (12), (13), (6), (7) and to use it for design purpose. Specifically, given the interval $\left[v_{1}, v_{2}\right]$ associated to the topology possible variations, we derive conditions on $A$ or $B C$ for MAS consensuability.

We consider a graph of $n=400$ and $k=4$. In the evolving network simulations, we started with $k=4$ and bounded it to the order of $O(\log (n))$ for setting a sparse graph. In Tab 1 are drawn the node dynamic and coupling matrices considered in the first set of simulations. 


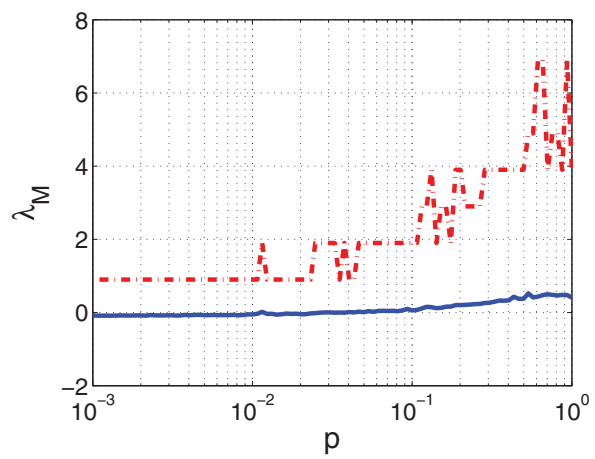

(a)

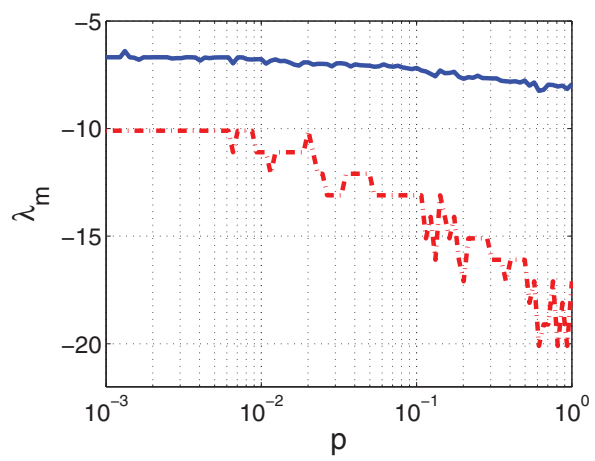

(c)

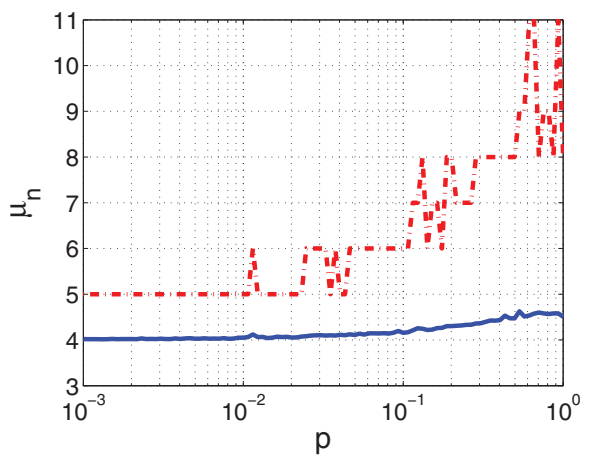

(b)

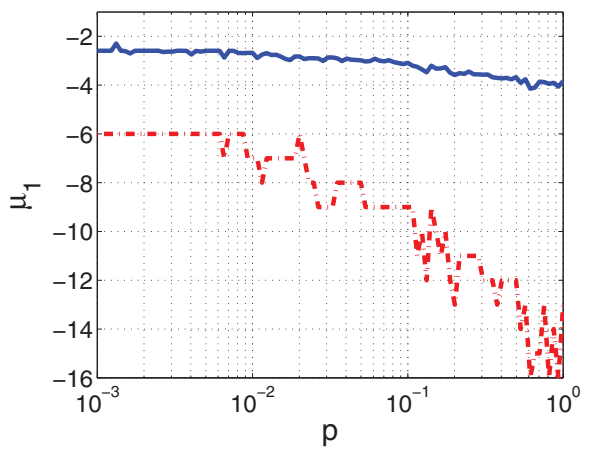

(d)

Fig. 2. Case 1. Dashed line: bound on the eigenvalue; continuous line: eigenvalues, (a) Maximum eigenvalue of $A_{g}$, (b) Maximum eigenvalue of $U$, (c) Minimum eigenvalue of $A_{g}$, (d) Minimum eigenvalue of $U$

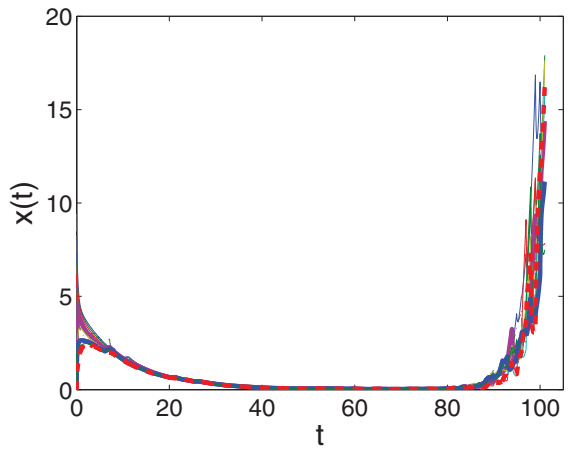

Fig. 3. Case 1: State dynamic evolution in the time 


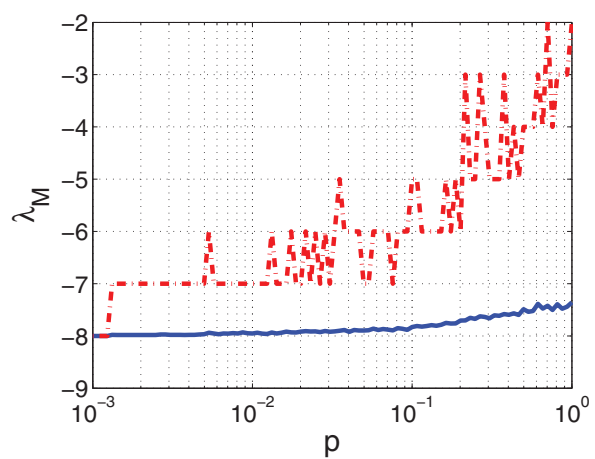

(a)

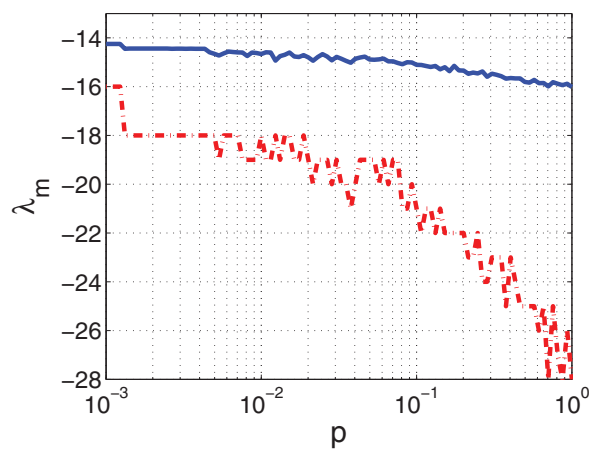

(c)

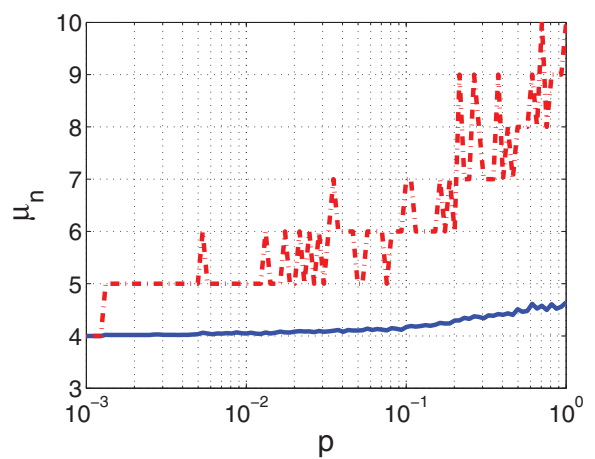

(b)

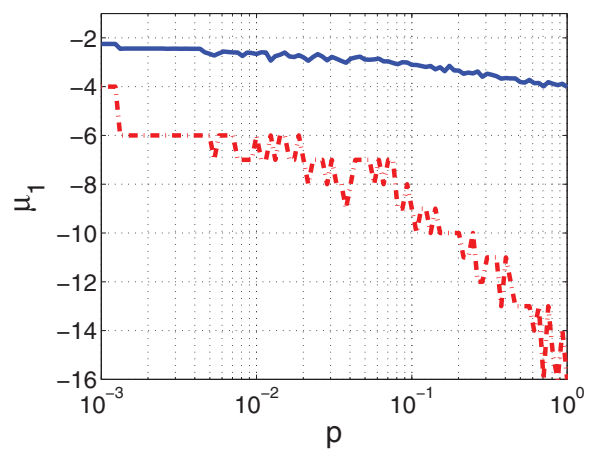

(d)

Fig. 4. Case 2. Dashed line: bound on the eigenvalue; continuous line: eigenvalue: (a) Maximum eigenvalue of $A_{g}$, (b) Maximum eigenvalue of $U$, (c) Minimum eigenvalue of $A_{g}$, (d) Minimum eigenvalue of $U$

\begin{tabular}{l||c|c|c}
\hline \hline & A & B & C \\
\hline \hline Case 1: & -4.1 & 1 & 1 \\
\hline Case 2: & -12 & 1 & 1 \\
\hline Case 3: & -6 & 1 & 1 \\
\hline Case 4: & -6 & 2 & 1 \\
\hline
\end{tabular}

Table 1 . Node system matrices $(A, B, C)$ 


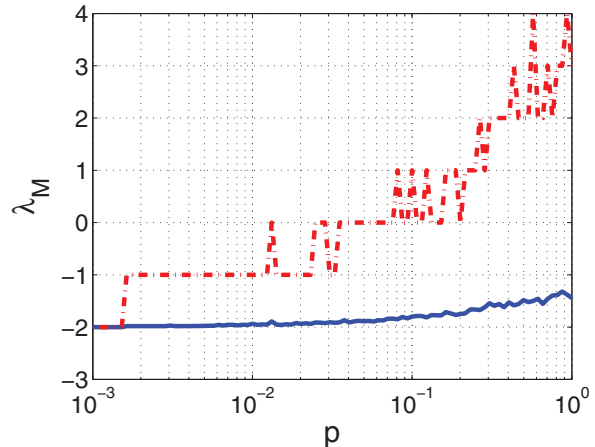

(a)

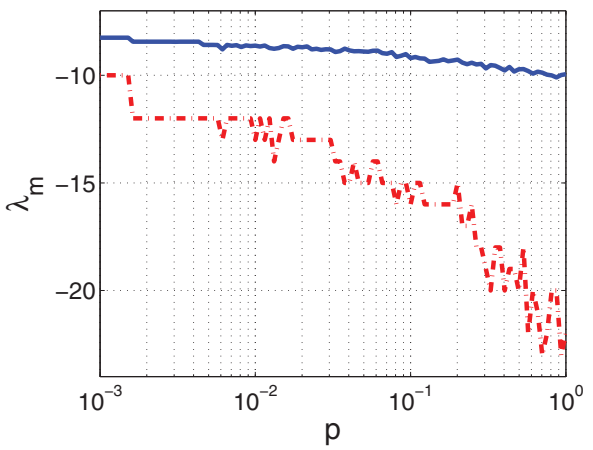

(c)

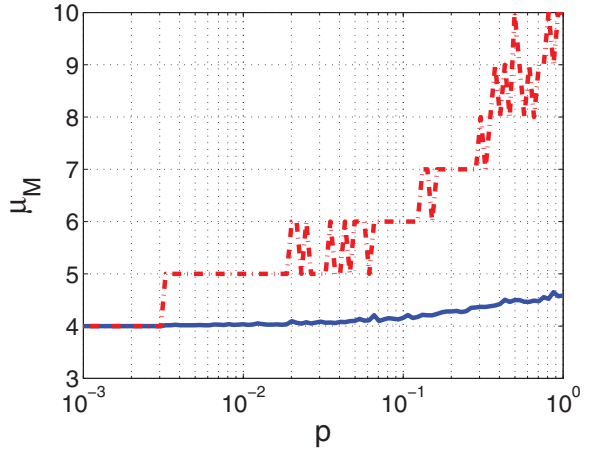

(b)

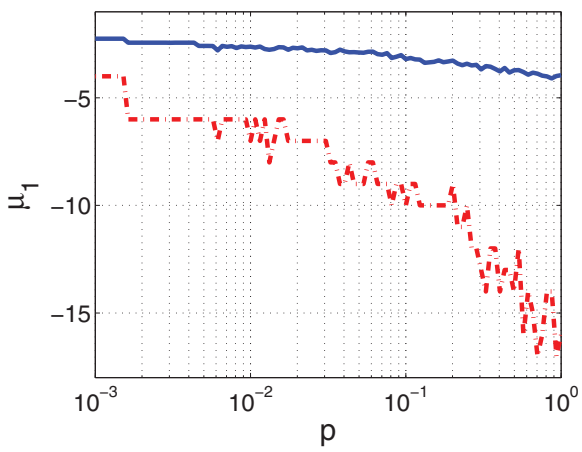

(d)

Fig. 5. Case 3. Dashed line: bound on the eigenvalue; continuous line: eigenvalue: (a) Maximum eigenvalue of $A_{g}$, (b) Maximum eigenvalue of $U$, (c) Minimum eigenvalue of $A_{g}$, (d) Minimum eigenvalue of $U$

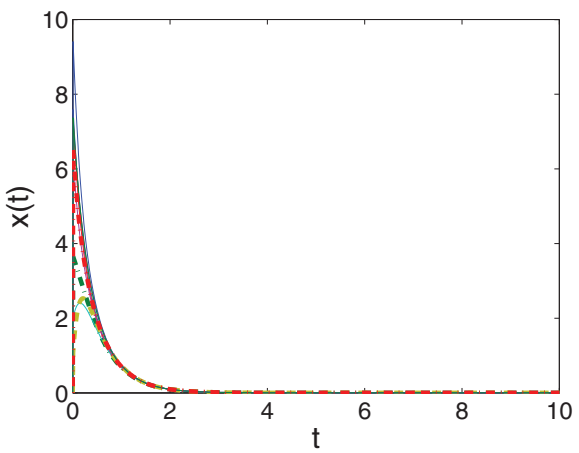

Fig. 6. Case 3: state dynamic evolution in the time 


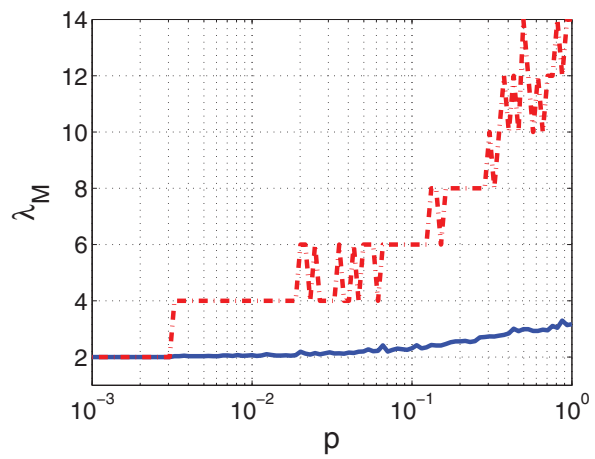

(a)

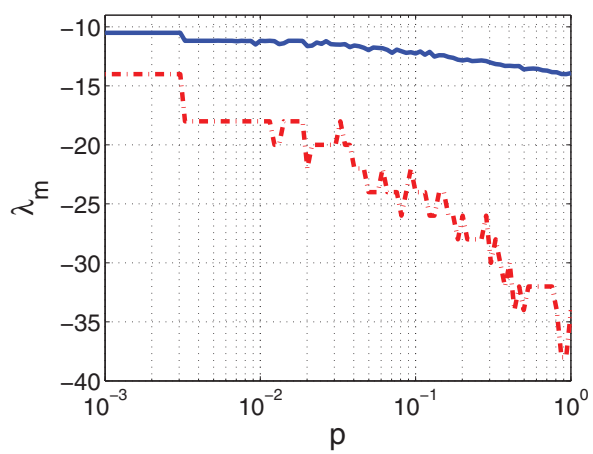

(c)

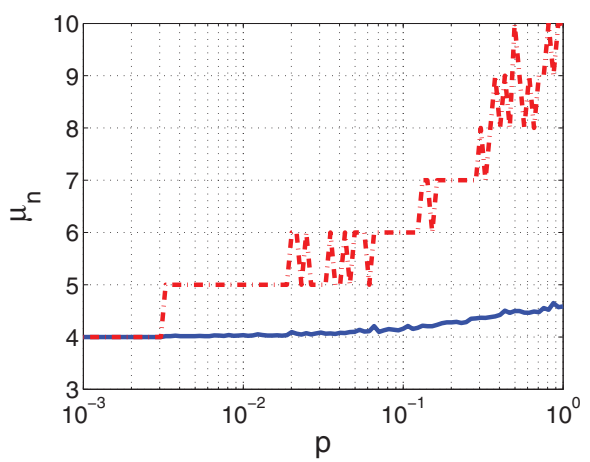

(b)

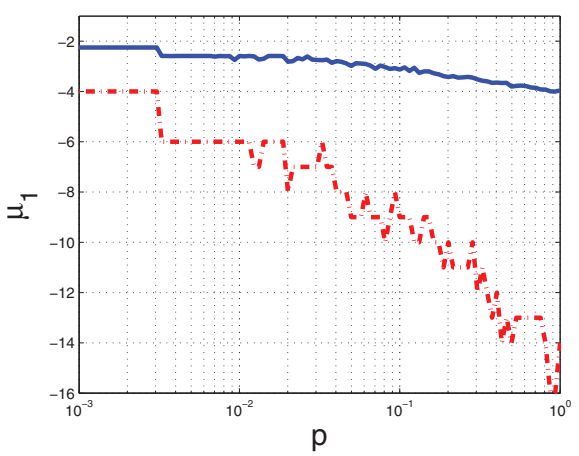

(d)

Fig. 7. Case 4. Dashed line: bound on the eigenvalues; continuous line: eigenvalues: (a) Maximum eigenvalue of $A_{g}$, (b) Maximum eigenvalue of $U$, (c) Minimum eigenvalue of $A_{g}$, (d) Minimum eigenvalue of $U$

In the case 1 (Fig 2), we note as although we start from a stable MAS network, the topology variation leads the network instability condition (namely $\lambda_{M}$ becomes positive). In Fig. 3 it is shown the time state evolution of the firsts 10 nodes, under the switching frequency of $1 \mathrm{~Hz}$. We note as the MAS converges to the consensus state till it is stable, then goes in instability condition.

In the case 2, we consider a node dynamic faster than the maximum network degree $d_{M}$ of all evolving network topologies from compete to random graph. Notice that although this assures MAS consensuability as drawn in Fig. 4, it can be much conservative.

In the case 3 (Fig 5), we consider a slower node dynamic than the cases 2 . The MAS is robust stable under topology variations. In Fig. 6 the state dynamic evolution is convergent and the settling time is about $4.6 /\left|\lambda_{M}\left(A_{g}\right)\right|$.

Then we have varied the value for $B C$ by doubling the $B$ matrix value leaving unchanged the node dynamic matrix. As appears in Fig. 7, the MAS goes in instability condition pointing out 


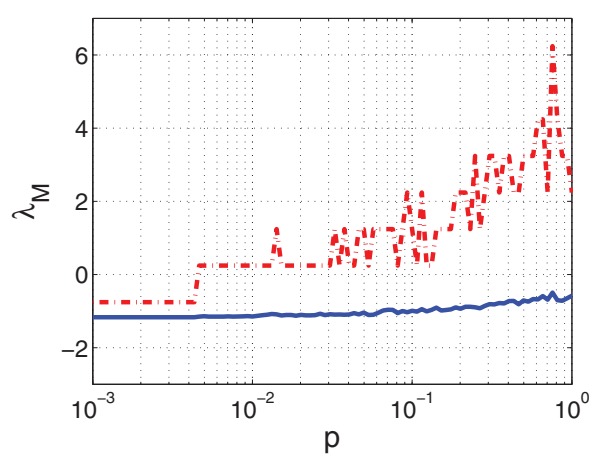

(a)

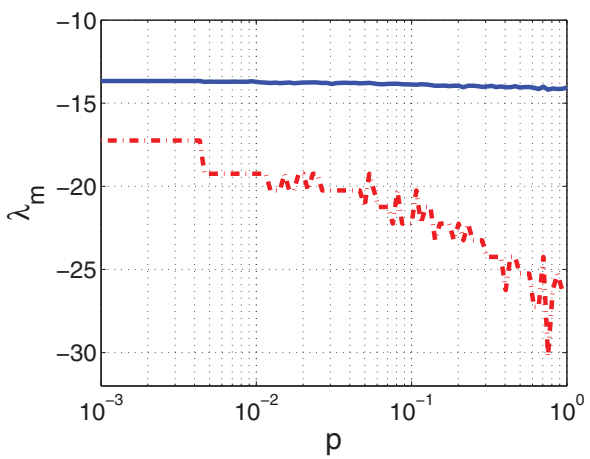

(c)

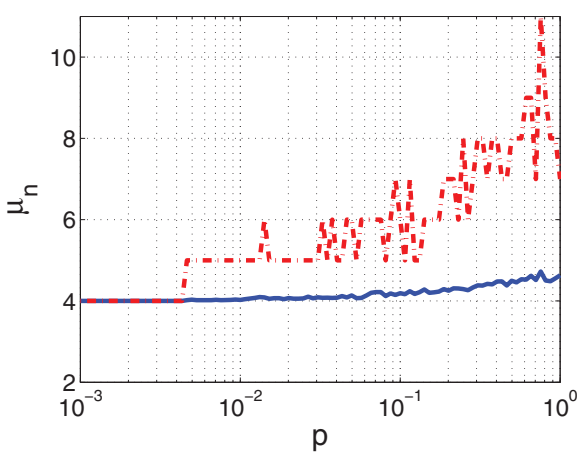

(b)

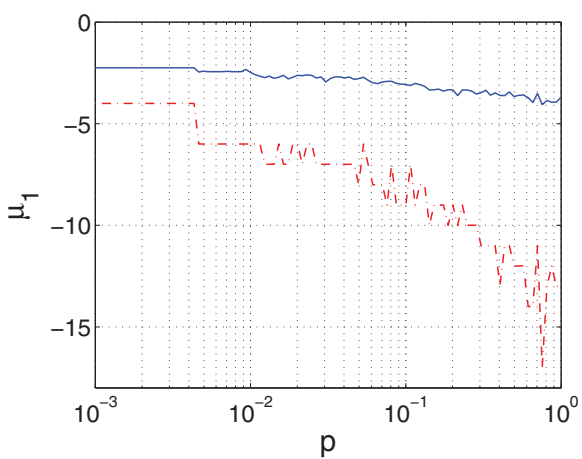

(d)

Fig. 8. Case 5. Dashed line: bound on the eigenvalue; continuous line: eigenvalue: (a) Maximum eigenvalue of $A_{g}$, (b) Maximum eigenvalue of $U$, (c) Minimum eigenvalue of $A_{g}$, (d) Minimum eigenvalue of $U$

that also the coupling strength can affect the stability (as stated by the conditions (8), (9)) and that this effect can be amplified by the network topological variations.

\begin{tabular}{c||c|c|c}
\hline \hline & $\mathbf{A}$ & $\mathbf{B}$ & $\mathbf{C}$ \\
\hline \hline Case 5: & {$\left[\begin{array}{cc}-6 & 3 \\
3 & -12\end{array}\right]$} & {$\left[\begin{array}{l}1 \\
0\end{array}\right]$} & {$\left[\begin{array}{ll}1 & 0\end{array}\right]$} \\
\hline Case 6: & {$\left[\begin{array}{cc}-3 & 3 \\
3 & -6\end{array}\right]$} & {$\left[\begin{array}{l}1 \\
0\end{array}\right]$} & {$\left[\begin{array}{ll}1 & 0\end{array}\right]$} \\
\hline Case 7: & {$\left[\begin{array}{cc}-3 & 3 \\
3 & -6\end{array}\right]$} & {$\left[\begin{array}{c}0.25 \\
0\end{array}\right]$} & {$\left[\begin{array}{ll}1 & 0\end{array}\right]$} \\
\hline
\end{tabular}

Table 2. Node system matrices $(A, B, C)$. 


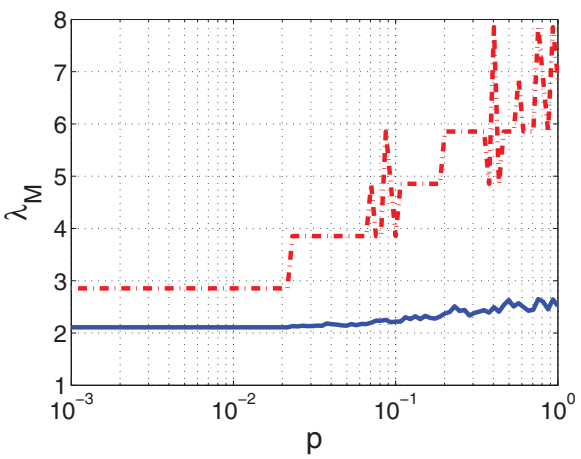

(a)

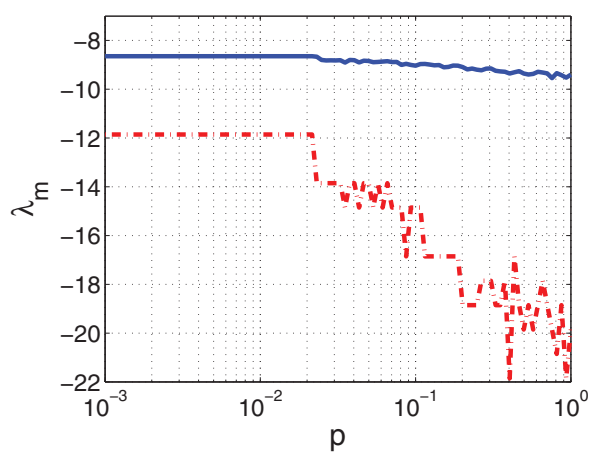

(c)

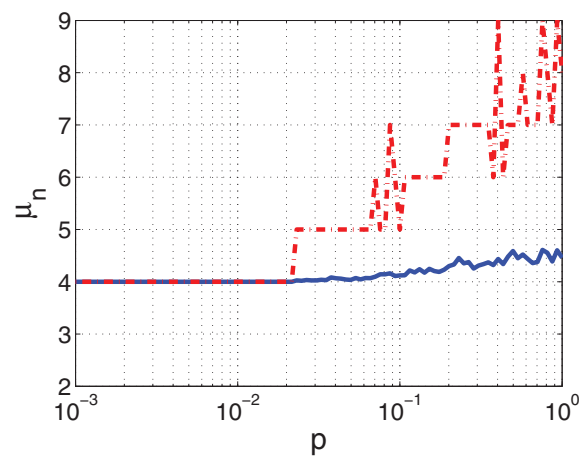

(b)

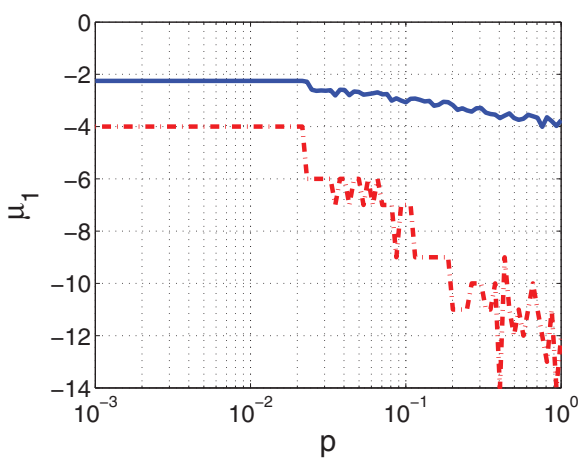

(d)

Fig. 9. Case 6. Dashed line: bound on the eigenvalue; continuous line: eigenvalue: (a) Maximum eigenvalue of $A_{g}$, (b) Maximum eigenvalue of $U$, (c) Minimum eigenvalue of $A_{g}$, (d) Minimum eigenvalue of $U$

On the other side, a reduction on $B C$ increases the MAS stability margin. So we can tune the $B C$ value in order to guarantee stability or desired robust stability MAS margin under a specified node dynamic and topology network variations. Indeed if $B C$ has eigenvalues above 1 , its effect is to amplify the eigenvalues of $U$ and we need a faster node dynamic for assessing MAS stability. If $B C$ has eigenvalues less of 1 , its effect is of attenuation and the node dynamic can be slower without affecting the network stability.

Now we consider SISO system of second order at the node as shown in Tab.2. In this case the matrix $B C$ has one zero eigenvalue being the rows linearly dependent.

In the case 5 the eigenvalues of $A$ are $\alpha_{1}=-4.76$ and $\alpha_{2}=-13.23$, the eigenvalues of the coupling matrix $B C$ are $v_{1}=1$ and $v_{2}=0$. In this case the node dynamic is sufficiently fast for guaranteeing MAS consensuability (Fig. 8). In the case 6, we reduce the node dynamic matrix $A$ to $\alpha_{1}=-1.15$ e $\alpha_{2}=-7.85$. Fig. 9 shows instability condition for the MAS network. We 


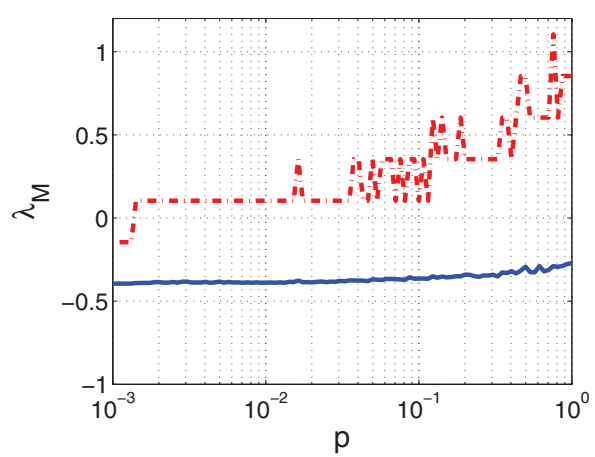

(a)

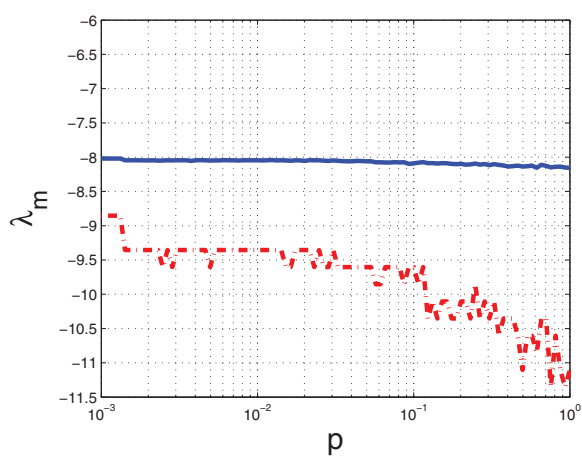

(c)

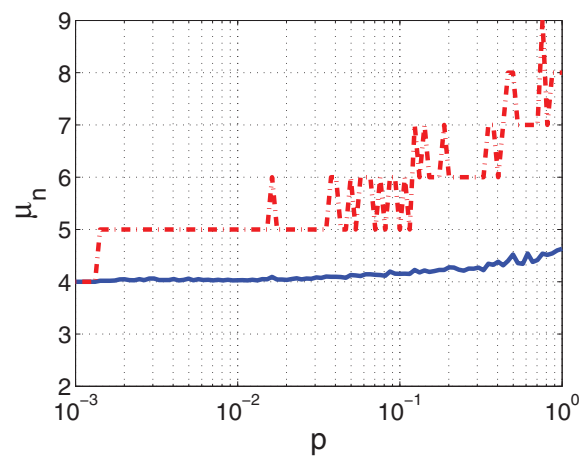

(b)

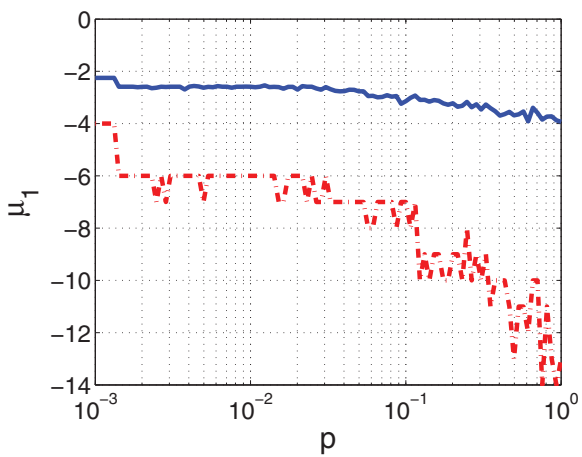

(d)

Fig. 10. Case 7. Dashed line: bound on the eigenvalue; continuous line: eigenvalue. (a) Maximum eigenvalue of $A_{g}$, (b) Maximum eigenvalue $U$, (c) Minimum eigenvalue of $A_{g}$, (d) Minimum eigenvalue of $U$

can lead the MAS in stability condition by designing the coupling matrix $B C$ as appear by the case 7 and the associate Fig. 10.

\subsection{Robustness to node fault}

Now we deal with the case of node fault. We can state the following Theorem.

Theorem 2 Let $A$ and $B C$ symmetric matrix and $G(V, E, U)$ an undirected graph. If the MAS system described by $A_{g}$ is stable, it is stable also in the presence of node faults. Moreover the MAS dynamic becomes faster after the node fault.

Proof Being the graph undirected and $A$ and $B C$ symmetric then $A_{g}$ is symmetric. Let $\tilde{A_{g}}$ the MAS dynamic matrix associated to the network after a node fault. $\tilde{A}_{g}$ is obtained from $A_{g}$ by eliminating the rows and columns corresponding to the nodes went down. So $\tilde{A}_{g}$ is a minor of $A_{g}$ and for the interlacing theorem (Horn R.A. \& Johnson C.R., 1995) it has eigenvalues inside 


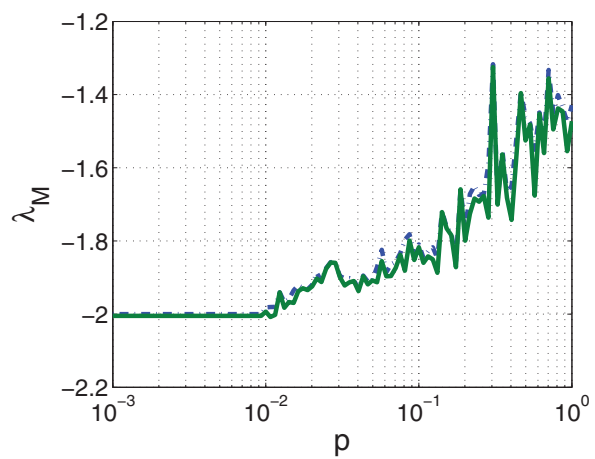

(a)

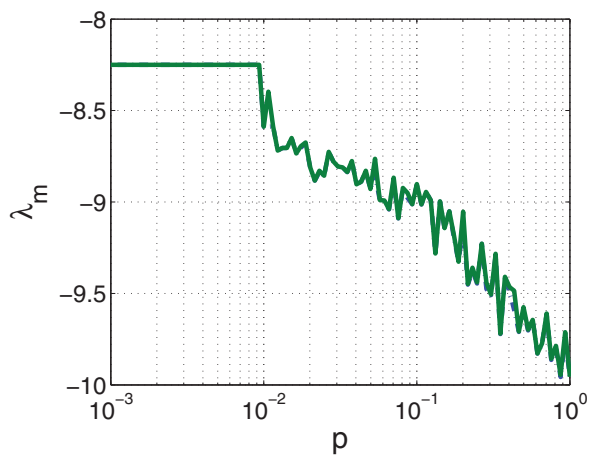

(c)

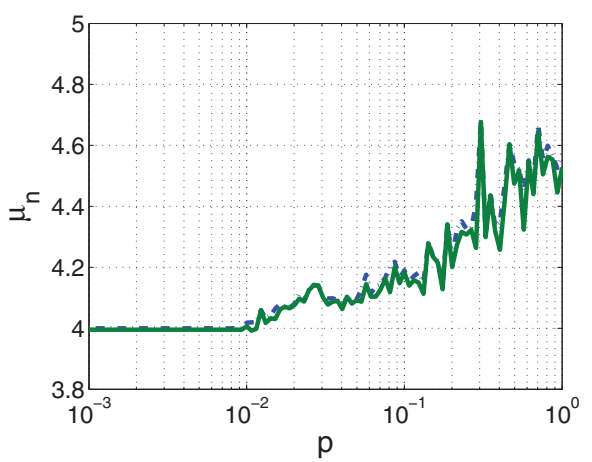

(b)

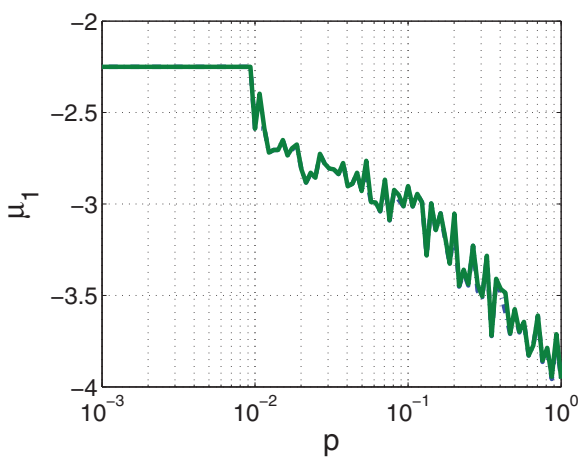

(d)

Fig. 11. Eigenvalues in the case $l=1$. Dashed line: eigenvalue in the case of complete topology with $n=100$; continuous line: eigenvalue in the case of node fault: (a) Maximum eigenvalue of $A_{g}$, (b) Maximum eigenvalue of $U$, (c) Minimum eigenvalue of $A_{g}$, (d) Minimum eigenvalue of $U$

the real interval with extremes the minimum and maximum $A_{g}$ eigenvalues. Hence if $A_{g}$ is stable, $\tilde{A_{g}}$ is stable too. Moreover, the maximum eigenvalue of $\tilde{A_{g}}$ is less than one of $A_{g}$. So the slowest dynamic of the system $\dot{x}(t)=\tilde{A_{g}} x(t)$ is faster than the system $\dot{x}(t)=A_{g} x(t)$.

In the follows we will show the eigenvalues of MAS dynamic in the presence of node fault. We consider MAS network with $n=100$. We compare for each evolving network topology at each time simulation step, the maximum and minimum eigenvalues of $A_{g}$ than those ones resulting with the fault of randomly chosen $l$ nodes. Figures 11 and 12 show the eigenvalues of system dynamic for the cases $l=1$ and $l=50$.

Notice that as the eigenvalues of $U$ and $A_{g}$ of fault network are inside the real interval containing the eigenvalues of $U$ and $A_{g}$ of the complete graph. In Fig. 13 are shown the time evolutions of state of the complete and faulted graphs. Notice that the fault network is faster than the initial network as stated by the analysis of the spectra of $A_{g}$ and $\tilde{A_{g}}$. 


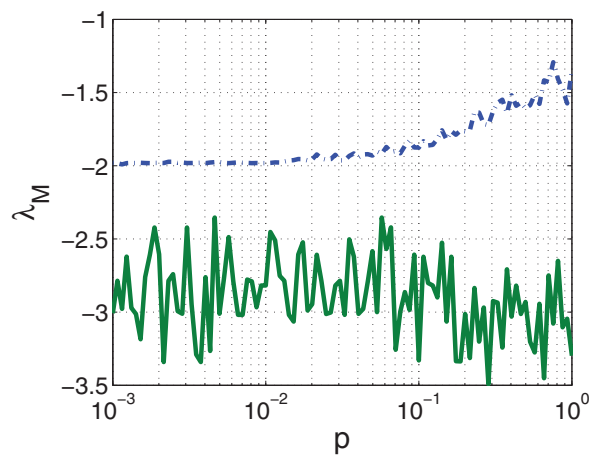

(a)

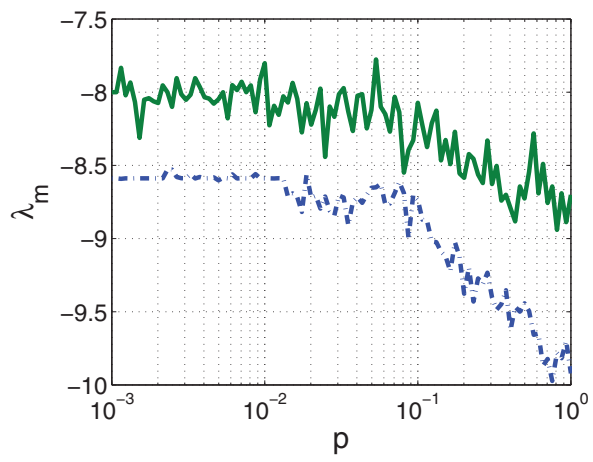

(c)

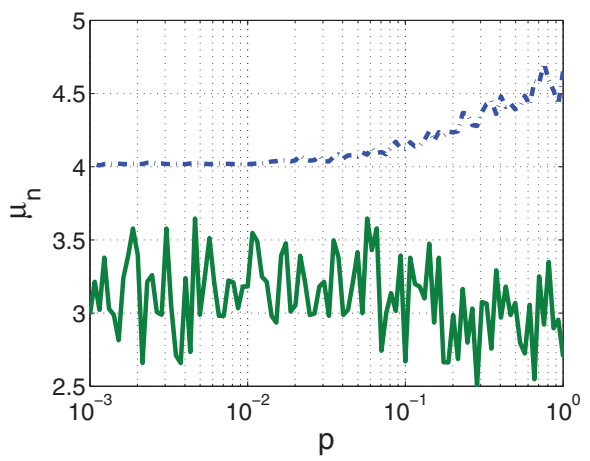

(b)

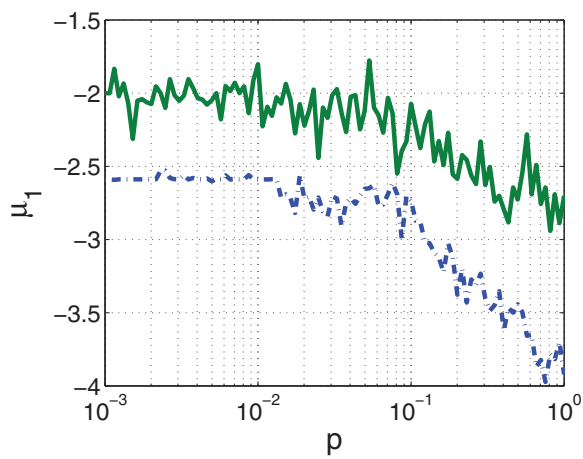

(d)

Fig. 12. Eigenvalues in the case of $l=50$. Dashed line: eigenvalue in the case of complete topology with $n=100$; continuous line: eigenvalue in the case of node fault: (a) Maximum eigenvalue of $A_{g}$, (b) Maximum eigenvalue of $U$, (c) Minimum eigenvalue of $A_{g}$, (d) Minimum eigenvalue of $U$

\section{Conclusions}

In this book chapter we have investigated the consensuability of the MASs under both the dynamic agent structure and communication topology variations. Specifically, it has given consensusability conditions of linear MASs as function of the agent dynamic structure, communication topology and coupling strength parameters. The theoretical results are given by transferring the consensusability problem to the stability analysis of LTI-MASs. Moreover, it is shown that the interplay among consensusability, node dynamic and topology must be taken into account for MASs stabilization: consensuability of MASs is assessed for all topologies, dynamic and coupling strength satisfying a pre-specified bound. From the practical point of view the consensuability conditions can be used for both the analysis and planning of MASs protocols to guarantee robust stability for a wide range of possible interconnection topologies, coupling strength and node dynamics. Also, the consensuability 

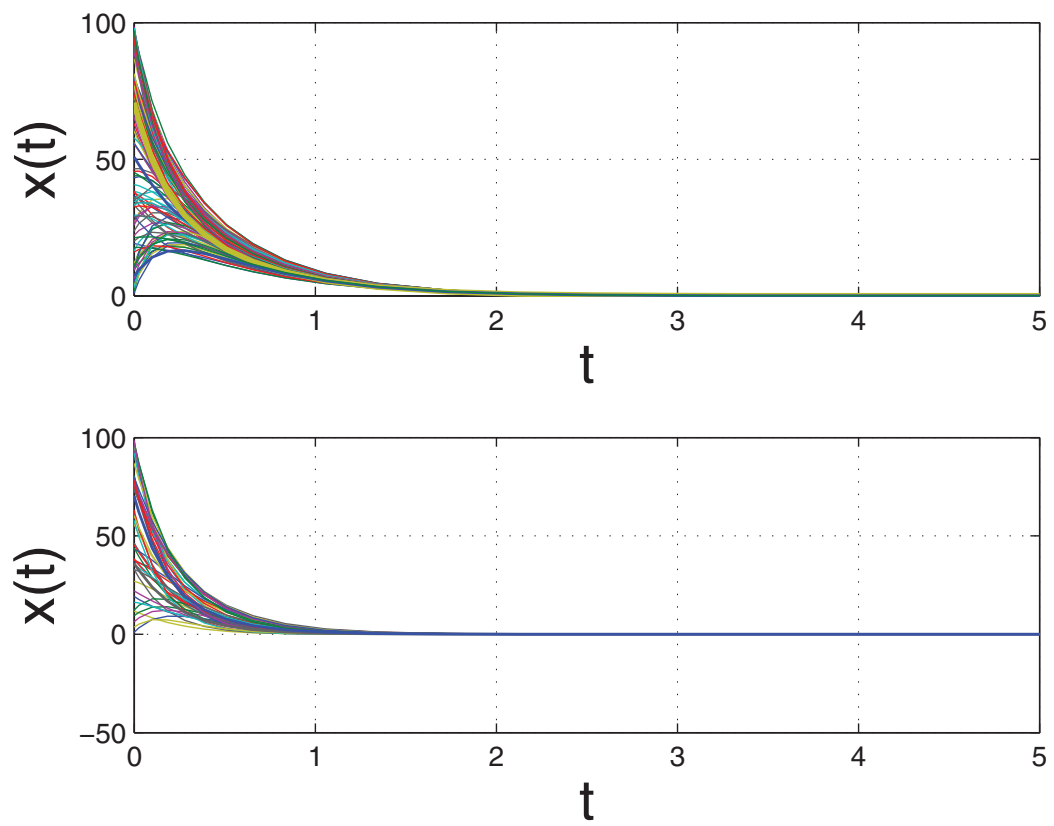

Fig. 13. Time evolution of the state variables for $1=50$ : top Figure: complete graph. Bottom Figure: graph with fault.

of MAS in the presence of node faults has been analyzed. Simulation scenarios are given to validate the theoretical results.

\section{Acknowledgement}

The author would like to thank Ms. F. Schioppa for valuable discussion.

\section{References}

J.K. Hedrick, D.H. McMahon, V.K. Narendran, and D. Swaroop. (1990). Longitudinal vehical controller design for IVHS systems. Proceedings of the American Control Conference, pages 3107-3112.

P. Kundur. (1994) Power System Stability and Control. McGraw-Hill.

D. Limebeer and Y.S. Hung. (1983). Robust stability of interconnected systems. IEEE Trans. Automatic Control, pages 710-716.

A. Michel and R. Miller. (1977). Qualitative analysis of large scale dynamical systems. Academic Press.

P.J. Moylan and D.J. Hill. (1978). Stability criteria for large scale systems. IEEE Trans. Automatic Control, pages 143-149.

F. Paganini, J. Doyle, and S. Low. (2001). Scalable laws for stable network congestion control. Proceedings of the IEEE Conference on Decision and Control, pages 185-190, 2001. 
M. Vidyasagar. (1977). L2 stability of interconnected systems using a reformulation of the passivity theorem. IEEE Transactions on Circuits and Systems, 24, 637-645.

D. ůSiljak. (1978). Large-Scale Dynamic Systems. Elsevier North-Holland.

J.C. Willems. (1976). Stability of large-scale interconnected systems..

Saber R.O., Murray R.M. (2004). Consensus Problems in Networks of Agents with Switching Topology and Time-Delays, IEEE Transactions on Automatic Control, Vol 49, 9.

Z. Lin, M. Brouke, and B. Francis, (2004). Local control strategies for groups of mobile autonomous agents., Transactions on Automatic Control, 49, vol 4, pages: 622Ú-629.

V. Blondel, J. M. Hendrickx, A. Olshevsky, and J. N. Tsitsiklis, (2005) Convergence in multiagent coordination, consensus, and flocking, 44th IEEE Conference on Decision and Control and European Control Conference, pages 2996Ü-3000.

A. V. Savkin, (2004) Coordinated collective motion of groups of autonomous mobile robots: analysis of Vicsek Šs model., Transactions on Automatic Control, Vol 49, 6, pages: 981-Ü982.

J. N. Tsitsiklis, D. P. Bertsekas, M. Athans, (1986). Distributed Asynchronous Deterministic and Stochastic Gradient Optimization Algorithms, Transactions on Automatic Control, pages. 803-Ü812.

C. C. Cheaha, S. P. Houa, and J. J. E. Slotine, (2009). Region-based shape control for a swarm of robots., Automatica, Vol. 45, 10, pages: 2406-Ü2411.

Ren, W., (2009). Collective Motion From Consensus With Cartesian Coordinate Coupling ., IEEE Transactions on Automatic Control. Vol. 54, 6, pages: 1330-1335.

S. E. Tuna, (2008). LQR-based coupling gain for synchronization of linear systems, Available at: http://arxiv.org/abs/0801.3390

Luca Scardovi, Rodolphe Sepulchre, (2009) Synchronization in networks of identical linear systems Automatica, Volume 45, Issue 11, Pages 2557-2562

W. Ren and R. W. Beard, (2005), Consensus seeking in multiagent systems under dynamically changing interaction topologies, IEEE Trans. Automatic Control, vol. 50, no. 5, pp. 655-661.

Ya Zhanga and Yu-Ping Tian, (2009). Consentability and protocol design of multi-agent systems with stochastic switching topology., Automatica, Vol. 45, 5, 2009, Pages 1195-1201.

R. Cogill, S. Lall, (2004). Topology independent controller design for networked systems, IEEE Conference on Decision and Control, Atlantis, Paradise Island, Bahamas, Dicembre 2004

D. J. Watts, S. H. Strogatz, (1998). Collective dynamics of small world networks, Nature - Macmillan Publishers Ltd, Vol. 393, Giugno 1998.

Horn R.A. and Johnson C.R., (1995). Topics in Matrix Analysis Cambridge University Press 1995. 


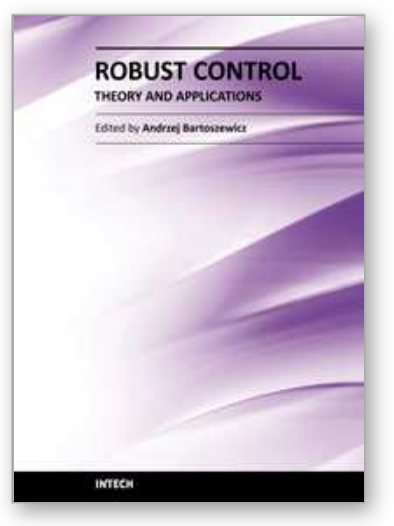

\author{
Robust Control, Theory and Applications \\ Edited by Prof. Andrzej Bartoszewicz
}

ISBN 978-953-307-229-6

Hard cover, 678 pages

Publisher InTech

Published online 11, April, 2011

Published in print edition April, 2011

The main objective of this monograph is to present a broad range of well worked out, recent theoretical and application studies in the field of robust control system analysis and design. The contributions presented here include but are not limited to robust PID, H-infinity, sliding mode, fault tolerant, fuzzy and QFT based control systems. They advance the current progress in the field, and motivate and encourage new ideas and solutions in the robust control area.

\title{
How to reference
}

In order to correctly reference this scholarly work, feel free to copy and paste the following:

Sabato Manfredi (2011). Consensuability Conditions of Multi Agent Systems with Varying Interconnection Topology and Different Kinds of Node Dynamics, Robust Control, Theory and Applications, Prof. Andrzej Bartoszewicz (Ed.), ISBN: 978-953-307-229-6, InTech, Available from:

http://www.intechopen.com/books/robust-control-theory-and-applications/consensuability-conditions-of-multiagent-systems-with-varying-interconnection-topology-and-differen

\section{INTECH}

open science | open minds

\section{InTech Europe}

University Campus STeP Ri

Slavka Krautzeka 83/A

51000 Rijeka, Croatia

Phone: +385 (51) 770447

Fax: +385 (51) 686166

www.intechopen.com

\section{InTech China}

Unit 405, Office Block, Hotel Equatorial Shanghai

No.65, Yan An Road (West), Shanghai, 200040, China

中国上海市延安西路65号上海国际贵都大饭店办公楼 405 单元

Phone: +86-21-62489820

Fax: $+86-21-62489821$ 
(C) 2011 The Author(s). Licensee IntechOpen. This chapter is distributed under the terms of the Creative Commons Attribution-NonCommercialShareAlike-3.0 License, which permits use, distribution and reproduction for non-commercial purposes, provided the original is properly cited and derivative works building on this content are distributed under the same license. 